The response of human muscles triceps brachii and vastus lateralis to training, consisting of 18.5 miles a day of skiing with a backpack, 6 days a week for 8 weeks, has been investigated by means of histochemical fiber typing (myofibrillar ATPase) as well as immunohistochemical discrimination of slow and fast myosin $(n=6)$. A detraining period of 33 weeks was also studied. The percentage of type 2 fibers decreased by $6 \%$ in the triceps brachii during training, whereas the intermediate fiber type increased by $4 \%$. No change was found in the distribution of fiber types in the vastus lateralis during training or in both muscles during detraining. The ATPase intermediate fibers also stained intermediately in the immunohistochemical stainings for slow and fast myosin. These results show that transformation of a fraction of the type 2 fibers into the intermediate type may occur as a response to endurance training.

MUSCLE \& NERVE 5:628-636

1982

\title{
TRAINING-INDUCED INCREASE IN MYOFIBRILLAR ATPaSE INTERMEDIATE FIBERS IN HUMAN SKELETAL MUSCLE
}

\author{
PETER SCHANTZ, MPE, RUDOLF BILLETER, PhD, \\ JAN HENRIKSSON, MD, PhD, \\ and EVA JANSSON, DrMedSc
}

The overwhelming number of longitudinal training studies performed to date on human and animal muscle provide, with few exceptions, ${ }^{25.34 .50}$ no evidence of interconversion between slow-twitch (type 1) and fast-twitch (type 2) muscle fibers. ${ }^{2,13,14,18.45 .51}$ The high percentage of type 1 fibers in the muscles of endurance athletes and the low percentage of this fiber type in sprinters ${ }^{8,15.22}$ have therefore been ascribed to genetic factors. The failure to observe a training-induced fiber type interconversion may, however, be due to the fact that no experiment has subjected a sufficiently untrained muscle to a sufficiently comprehensive and protracted training program. This explana-

From the Department of Physiology III, Karolinska Institute (Mr. Schantz. Dr. Henriksson), the Department of Clinical Physiology, Karolinska Sjukhuset, Stockholm. Sweden (Dr. Jansson), and the Institute of Pharmacology and Biochemistry. University of Zürich, Zürich, Switzerland (Dr. Billeler).

Acknowledgments: We wish to thank Ms. A. V. Schewelow and U. Siltberg for excellent technical and secretarial assistance, respectively. and Professor T. Caspersson for placing the microphotometer at our disposal. This study was supported by grants from the Research Council of the Swedish Sports Federation and from the Swedish National Defense Research Institute.

Address reprint requests to Mr. Schantz, at the Department of Physiology III, Karolinska Instilutet, Lidingövägen 1, S-114 33 Stockholm, Sweden.

Received for publication January 5, 1982; revised manuscripl accepted for publication July 7, 1982.

0148-639x/0508/0628 $\$ 1.25 / 0$

C 1982 John Wiley \& Sons, Inc tion is supported by the studies of Jansson and co-workers, ${ }^{24}$ who noted a decrease in type 1 fibers (17\%) with a concomitant increase in fibers staining intermediately with myofibrillar adenosine triphosphatase (ATPase) stain (11\%), when a group of distance runners changed its training regimen from protracted running at lower intensities to a running program at higher intensities but with shorter duration. This intermediately staining fiber type, which has been termed type $2 \mathrm{C}$, " "unclassified," 44 or type $2 \mathrm{C}-1 \mathrm{~B}^{11}$ may well be a transitional stage between type 1 and type 2 .

The aim of the present study was to investigate whether an untrained muscle (triceps brachii) and a postural and therefore relatively trained muscle (quadriceps femoris, vastus lateralis) in humans would respond to extensive endurance training of very long duration with a type 2 to type 1 interconversion and/or with an increased proportion of intermediate type fibers. For this purpose, we employed both histochemical and immunohistochemical techniques.

\section{MATERIALS AND METHODS}

Subjects. Six healthy men, average age 25 years, (range 23 to 30 ), height $183 \mathrm{~cm}$ (175 to 197), and weight $76 \mathrm{~kg}$ (67 to 84 ), participated in this study. They were informed about the procedure and risks involved in the experiments before they volunteered. The study was approved by the Com- 


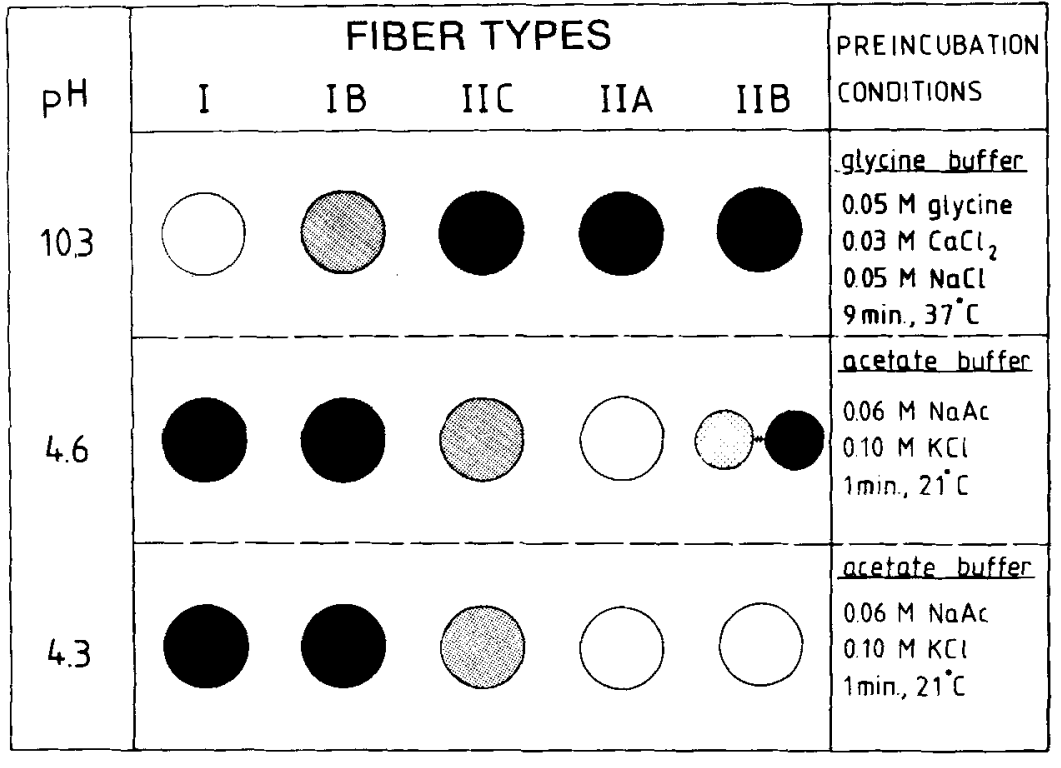

Figure 1. Staining characteristics for the different fiber types. (The $\mathrm{pH}$ of the acetate buffer was adjusted to 4.3 and 4.6 with acetic acid. The $\mathrm{pH}$ of the glycine buffer was adjusted to 9.4 and 10.3 using hydrochloric acid and sodium hydroxide, respectively.) mittee of Ethics at Karolinska Institutet. The subjects had performed regular physical exercise 2 to 5 times for about 40 minutes per week, mainly involving the legs (running, bicycling, cross-country skiing) for several years prior to the study. However, due to injuries and illnesses, only 2 subjects had been training regularly in the months immediately preceding the study. No subject had performed any arm training in the 8 months prior to the study. The triceps brachii muscle could therefore be characterized as truly untrained for the type of work it was going to perform. Normal training, such as running and bicycling, was recommenced during the detraining period. No specific arm training was performed during the detraining. All subjects had sedentary occupations.

Training. The training consisted of about 930 miles $(1,500 \mathrm{~km})$ of skiing with backpacks $(25 \mathrm{~kg})$ in the Swedish mountains. The distance was covered in 50 effective days of skiing, subdivided into 8 stages of about 6 days each, with 1 day of rest in between. The group thus skied about 18.5 miles per day, using about 5.5 hours of effective exercise each day.

Muscle Sampling. Muscle biopsies for histochemistry, immunohistochemistry, and enzymatic analysis were obtained from the middle portion of the lateral head of the quadriceps femoris (vastus lateralis-VL) in both the left and right leg and from the middle-lower portion of the medial head of the left triceps brachii (TB) using the needle-biopsy technique. ${ }^{3}$ Sampling from all 6 subjects was performed before training (week 0), after the third week of training (week 3), immediately after training (week 8), and at 6 weeks of detraining (week 14). Additional muscle samples were taken from the arms at 33 weeks of detraining (week 41). Sampling was performed around 48 hours (week $3 ; 24$ hours) after the last exercise session. No attempt was made to standardize the sampling with respect to the time of day.

Histochemical and Immunohistochemical Methods. The samples were immediately mounted in an embedding medium O.C.T. compound (Tissue-Tek II, Lab-Tek Products, Naperville, IL) and frozen in isopentane, which was cooled in liquid nitrogen and stored at $-80^{\circ} \mathrm{C}$ until analysis. Serial transverse sections $(10 \mu \mathrm{m})$ were cut with a microtome at $-20 \mathrm{C}$ and stained for myofibrillar ATPase, ${ }^{17,36}$ NADH-tetrazolium reductase (NADH-TR) ${ }^{35}$ mitochondrial $\alpha$-glycerophosphate dehydrogenase $(\alpha-G P D H)^{52}$ activities, as well as with the amylasePAS method for visualizing capillaries ${ }^{1}$ and with an immunohistochemical method for demonstrating different myosin types. Sections to be stained for myofibrillar ATPase were preincubated at different $\mathrm{pHs}$ in acid (acetate) or alkaline (glycine) buffers as described in Figure 1.

The fibers were classified on the basis of their staining intensity (myofibrillar ATPase) into fiber types $\mathrm{l}$ and $2,{ }^{12}$ and into the subgroups $2 \mathrm{~A}$ and $2 \mathrm{~B},{ }^{6}$ and type $2 \mathrm{C}^{5,9}$ and $1 \mathrm{~B},{ }^{11}$ as shown in Figure 1. Two biopsies from each subject at each sampling 
were generally used for the fiber typing. Type $2 \mathrm{C}$ and $1 \mathrm{~B}$ fibers will henceforth be referred to as intermediate fibers, except in the immunohistochemical section, where type $2 \mathrm{C}$ and $1 \mathrm{~B}$ are discussed separately.

In our investigation type-specific antirabbit myosin sera prepared from guinea pigs were used. It has been previously shown that our antirabbit slow myosin (anti-SM) and antirabbit fast myosin (anti-FM) sera are specific to their homologous antigens and do cross-react with human myosins. ${ }^{4,30}$ Antirabbit heart myosin light chain (antiheart-LC) serum cross-reacts with the light chains of slow myosins but not with fast myosins obtained from various species, such as rabbit and human. Therefore, this serum serves as an excellent marker for slow-type myosin light chains. Immunocytochemical detection of myosin types was performed on sections essentially as described by Billeter and co-workers. ${ }^{4}$ The procedure was modified insofar as the peroxidase-antiperoxidase complex $\left(\mathrm{PAP}^{49}\right)$, was chosen to visualize the reaction between antimyosin and myosin. In previous studies, a protein A-peroxidase conjugate was used. ${ }^{4.31}$ However, the PAP complex allows higher serum dilutions. The dilutions of antisera were anti-SM $1: 20$ or $1: 30$, anti-FM $1: 500$ or $1: 1000$, and antiheart-LC 1:100 in phosphate buffer solution (PBS), $(\mathrm{NaCl} 0.14 \mathrm{M}, \mathrm{KCl} 0.003 \mathrm{M}, \mathrm{Na}-$ phosphate $0.01 \mathrm{M}, \mathrm{pH} 7.3$ ) and rabbit antiguinea pig IgG (Cappel Lab. Inc., Cochranville, PA) 1:50, PAP (for example, guinea pig, Cappel) $1: 50$, in serum-PBS (rabbit nonimmune diluted 1:20 in PBS).

Rating the Intensity of Immunohistochemical, Myofibrillar ATPase, Oxidative, and Glycolytic Staining. The staining intensity of single fibers in the immunohistochemical and the myofibrillar ATPase stainings was examined microphotometrically. It soon became evident that it was easy, on the basis of microscopic inspection, to predict the rating made by the apparatus. Since the microphotometric rating was very time-consuming, the remainder of the sections were therefore examined subjectively. The microphotometric determinations were performed using an M.P.V. microphotometer (Leitz, Wetzlar, West Germany). The measuring field was $200 \mu \mathrm{m}^{2}$ in the center of the fiber. The transmission of light through the immunohistochemical (450 nm) and myofibrillar ATPase $(470 \mathrm{~nm})$ stainings was measured in relation to the transmission through the slide, the mounting medium and cover glass just outside the section. Subjective ratings were performed independently by 2 persons on a scale from 1 (light) to 5 (dark). Mean values were employed in the few cases when the ratings differed. The mean values for light transmission through the different fiber types were also converted according to this scale. A subjective rating of the oxidative (NADH-TR) and glycolytic ( $\alpha$-GPDH) capacity of the intermediate and types 1 and 2 fibers in the TB at week 8 and week 41 was carried out as described. The type 1 and type 2 fibers stained homogeneously in both stainings and were rated grade 3 (dark) or 1 (light).

Area Measurements. Sections stained with the amylase-PAS method were used in measuring the cross-sectional areas of the muscle fibers. The measurements were made using a grid method. ${ }^{10}$ The emphasis in this paper with respect to area results is to compare each subject's intermediate fiber type area in relation to the area of types $1,2 \mathrm{~A}$, and $2 \mathrm{~B}$ fibers.

Other area results will be published separately. ${ }^{46}$

Number of Samples and Fibers Included in the Determination of Staining Intensity and Cross-sectional Area of Intermediate Fibers. In general, the aim has been to include as many samples as possible in the determinations. However, in some samples the number of intermediate fibers suited to determination was too low to permit any satisfactory comparison. All of 16 samples (mainly from week 8 and week 14; 8 from TB and VL, respectively) from 4 subjects were examined in a random sequence. From each sample about 20 fibers each of fiber types $1,2 \mathrm{~A}$, and $2 \mathrm{~B}$, as well as about 10 intermediate fibers, were examined or rated. A total of 11 samples from 5 subjects' TB at weeks 8 and 41 were rated. All of 12 samples from 5 subjects constituted the basis for the comparison of the intermediate fibers to the other fiber types. The area of about 40 fibers of each fiber type 1,2A, and $2 \mathrm{~B}$, as well as about 10 intermediate fibers from each sample was measured.

Statistics. Results in the text and tables concerning fiber type distribution are given as median value: and range. Other results will be given as means and ranges or standard error of mean (SEM). A twoway analysis of variance (ANOVA) of no difference in means was applied to the data on fiber type distribution. When the ANOVA indicated an overall significance between the sampling occasions, comparisons among means ${ }^{47}$ were applied. 


\begin{tabular}{|c|c|c|c|c|c|}
\hline \multirow[b]{2}{*}{ Fiber type } & \multicolumn{3}{|c|}{ Training } & \multicolumn{2}{|c|}{ Detraining } \\
\hline & Week 0 & Week 3 & Week 8 & Week 14 & Week 41 \\
\hline 1 & $\begin{array}{c}42.7 \\
(20-62)\end{array}$ & $\begin{array}{l}48.7 \\
(20-77)\end{array}$ & $\begin{array}{l}45.2 \\
\quad(26-67)\end{array}$ & $\begin{array}{l}46.9 \\
(31-78)\end{array}$ & $\begin{array}{l}45.6 \\
(22-76)\end{array}$ \\
\hline $2 A$ & $\begin{array}{c}53.4 \\
(38-59)\end{array}$ & $\begin{array}{l}50.5 \\
(23-51)\end{array}$ & $\begin{array}{l}49.6 \\
(28-54)\end{array}$ & $\begin{array}{l}33.8^{\text {a.th.c }} \\
(12-48)\end{array}$ & $\begin{array}{l}342^{4} \\
(22-48)\end{array}$ \\
\hline $2 \mathrm{~B}$ & $\begin{array}{c}0.5 \\
(0-34)\end{array}$ & $\begin{array}{l}0.6 \\
(0-24)\end{array}$ & $\begin{array}{l}1.2 \\
(0-8)\end{array}$ & $\begin{array}{l}7.9 \\
(0-26)\end{array}$ & $\begin{array}{l}9.4 \\
(0-34)\end{array}$ \\
\hline $2 A+2 B$ & $\begin{array}{c}57.3 \\
(38-80)\end{array}$ & $\begin{array}{l}51.0 \\
(23-73)\end{array}$ & $\begin{array}{l}52.8^{4} \\
\quad(28-61)\end{array}$ & $\begin{array}{l}49.5^{a} \\
(12-57)\end{array}$ & $\begin{array}{l}52.4^{a} \\
(22-62)\end{array}$ \\
\hline Intermediate & $\begin{array}{c}0.0 \\
(0-0.4)\end{array}$ & $\begin{array}{l}0.4 \\
(0-7)\end{array}$ & $\begin{array}{l}4.0 \\
(0.2-17)\end{array}$ & $\begin{array}{l}5.2 \\
(0-12)\end{array}$ & $\begin{array}{l}4.5 \\
(0-15)\end{array}$ \\
\hline $\begin{array}{l}\text { Number of } \\
\text { fibers } \\
\text { counted }\end{array}$ & $\begin{array}{c}243 \\
(95-417)\end{array}$ & $\begin{array}{c}394 \\
(150-753)\end{array}$ & $\begin{array}{c}507 \\
(241-619)\end{array}$ & $\begin{array}{c}315 \\
(174-448)\end{array}$ & $\begin{array}{c}418 \\
(312-545)\end{array}$ \\
\hline
\end{tabular}

Fiber type distribution in the triceps brachii (medians and ranges). The letters a, $b$, and $c$ designate significant differences $(P<0.05)$ compared to weeks 0,3 , and 8 , respectively. In the pretraining state intermediate fibers (type $2 \mathrm{C}$ and type (B) were only seen in 1 subject, so there was no basis for ANOVA. However, the proportion of intermedrate fibers was greater in all subjects after 8 weeks of training than in pretraining and after 3 weeks of training. The population of type $2 B$ fibers was askew, so no ANOVA could be performed. No pattern of change was seen for type 28 .

Only differences with a significance probability level of $<0.05$ has been designated in figures and tables or commented on in the text. Otherwise this has been stated. The intraindividual differences in area of types $1,2 \mathrm{~A}$, and the intermediate type fibers were compared using paired Student's $t$ tests. ${ }^{47}$ In that test, the intermediate fibers were used twice to form intraindividual differences, which is why a higher significance level $(P<0.01)$ was chosen.

\section{RESULTS}

Fiber Type Distribution. Triceps brachii. During the 8 weeks of training, there was no significant change in either of the type 1 or $2 \mathrm{~A}$ percentages (median values and ranges given in Table 1 ). The population of type $2 \mathrm{~B}$ fibers was too askew to permit any statistical evaluation. However, when the type 2 fibers (type $2 \mathrm{~A}+2 \mathrm{~B}$ ) were counted together, a $6 \%(-3$ to -23$)$ decrease $(P<0.05)$ in absolute terms was noted. At pretraining, myofibrillar ATPase intermediate fibers were only found in one subject. An increase in the percentage of intermediate fibers was seen in all individuals (median $4.0 \%$, range 0.2 to 17.4 ) during training. During the first 6 weeks of detraining the type $2 \mathrm{~A}$ percentage decreased 15\% (3.4 to -22.8) $(P<0.05)$. No change was noted for either type 1 or the intermediate fiber type during detraining. After 33 weeks of detraining, both the type $2 \mathrm{~A}$ and 2 (A + B) percentage differed $(P<0.05) 14 \%(8$ to 28$)$ and $8 \%$ (4 to 28 ), respectively, as compared to the pretraining status, and intermediate fibers were still seen in 5 out of 6 subjects $(4.5 \%, 0.0$ to 15.4$)$.

Vastus lateralis. During the 8 weeks of training as well as during 6 weeks of detraining, there was no change in the proportion of any fiber type in the vastus lateralis (VL) (median values and ranges given in Table 2).

Immunohistochemical Staining Intensity of the Ditferent Fiber Types. The type 1 and type 2 ( $A$ and $B$ ) fibers in both the $\mathrm{TB}$ and VL displayed distinct staining intensity patterns. Type 2 fibers stained darkly with anti-FM and lightly with anti-SM and anti-HLC, whereas the type 1 fibers displayed a reciprocal pattern of staining intensity. On the basis of the staining for myofibrillar ATPase, intermediate fibers could be subdivided into type 2C and $1 \mathrm{~B}$ fibers (see Materials and Methods). They also stained in different and rather distinct patterns when the immunohistochemical method was employed. Type 1B fibers stained nearly as darkly with anti-SM and anti-HLC as the type 1 fibers, but displayed intermediate staining with anti-FM. Type 2C fibers stained nearly as darkly as type 2 fibers with anti-FM, but stained intermediately with anti-SM and anti-HLC (Fig. 2). In the VL, the staining pattern in $1 \mathrm{~B}$ and $2 \mathrm{C}$ varied somewhat more than in the TB. Furthermore, in some rarely occurring fibers in the VL, the ATPase staining intensity after alkaline preincubation was between 
Table 2. Muscle fiber type distribution (\%) in m. quadriceps femoris, vastus lateralis.

\begin{tabular}{lcccc}
\hline & & Training & & Detraining \\
\cline { 2 - 5 } Fiber type & Week 0 & Week 3 & Week 8 & Week 14 \\
\hline 1 & 58.0 & 53.0 & 53.0 & 52.2 \\
& $(27-66)$ & $(29-69)$ & $(32-69)$ & $(40-68)$ \\
$2 \mathrm{~A}$ & 35.2 & 32.0 & 29.2 & 30.4 \\
& $(24-42)$ & $(25-38)$ & $(20-41)$ & $(27-35)$ \\
$2 \mathrm{~B}$ & 10.1 & 14.2 & 13.7 & 12.8 \\
& $(0-30)$ & $(2-24)$ & $(3-32)$ & $(2-24)$ \\
$2 \mathrm{~A}+2 \mathrm{~B}$ & 41.4 & 46.7 & 46.6 & 45.8 \\
& $(32-71)$ & $(29-62)$ & $(31-62)$ & $(32-55)$ \\
Intermediate & 1.6 & 1.1 & 0.8 & 1.2 \\
& $(0.1-6)$ & $(0-9)$ & $(0.2-6)$ & $(0.2-6)$ \\
\hline \hline Number of & 1260 & 1288 & 1402 & 1040 \\
fibers & $(870-1630)$ & $(887-1434)$ & $(847-1549)$ & $(844-1907)$ \\
counted & & & & \\
\hline
\end{tabular}

Fiber type distribution in the quadriceps femoris, vastus lateralis (medians and ranges). No significant changes in fiber type distribution were noted.

that of type $1 \mathrm{~B}$ and type 1 . These fibers stained in the same way as type $l$ in the immunohistochemical stainings.

Oxidative and Glycolytic Staining Intensity of Intermediate Fibers Compared to Other Fiber Types. The staining intensities of the different fiber types in staining for oxidative (NADH-TR) and glycolytic $(\alpha-\mathrm{GPDH})$ capacity are shown in Figure 3 . After 8

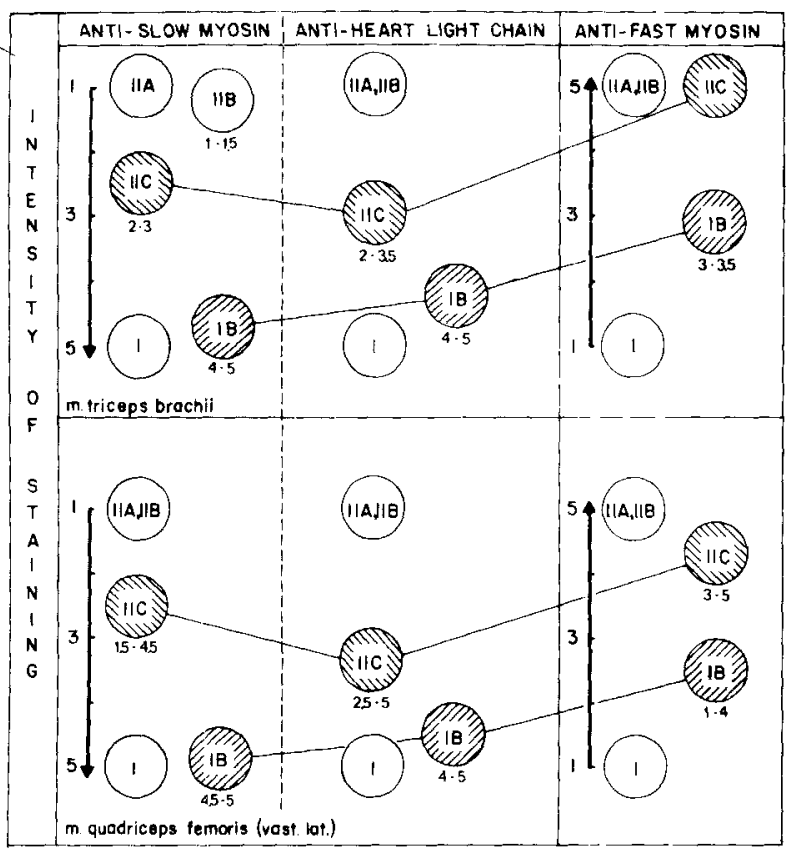

Figure 2. Immunohistochemical stainings toward slow and fast myosin as well as toward heart myosin light chain in the different fiber types. Fibers were rated from 1 (light) to 5 (dark). The lines connecting the circles representing fiber types $2 \mathrm{C}$ and $1 B$ only serve a didactic purpose. (Means and ranges.) weeks of training the staining of intermediate fibers in the TB was similar to that of type 1 fibers. During the following 33 weeks of detraining (week $41)$, the glycolytic staining intensity of the intermediate fibers increased, whereas the oxidative staining intensity decreased in 3 of the 5 subjects.

\section{Area of Intermediate Fibers Compared to Other Fiber}

Types. When each subject's fiber type 1 and $2 \mathrm{~A}$ areas in TB were compared to the area of the intermediate fibers, it was found that the area of type 1 was on the average $19.1 \% \pm 4.0(P<0.001)$ smaller, and that of type $2 \mathrm{~A}$ was $22.8 \% \pm 6.0(P<$ 0.01 ) larger (Fig. 4). The mean fiber type areas amounted to $5,900 \pm 380 \mu^{2}$ (type 1), $8,940 \pm$ $530 \mu \mathrm{m}^{2}$ (type $2 \mathrm{~A}$ ), and 7,700 $\pm 650 \mu \mathrm{m}^{2}$ (intermediate fibers). Due to the absence of type $2 \mathrm{~B}$ in many samples, this type could only be compared to

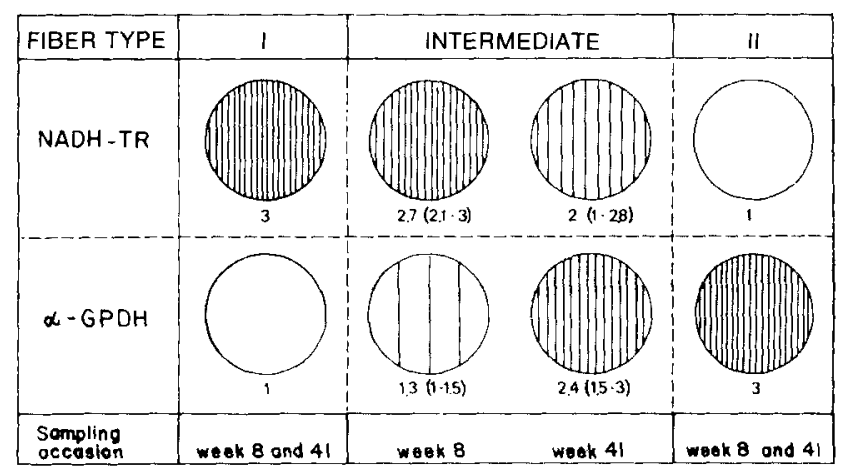

Figure 3. Stainings for oxidative (NADH-TR) and glycolytic capacity $(\alpha-G P D H)$ in types 1,2 , and intermediate fibers from $m$ triceps brachil after training (week 8) and at 33 weeks of detraining (week 41). Fibers were subjectively rated from 1 (light) to 3 (dark). (Means and ranges.) 


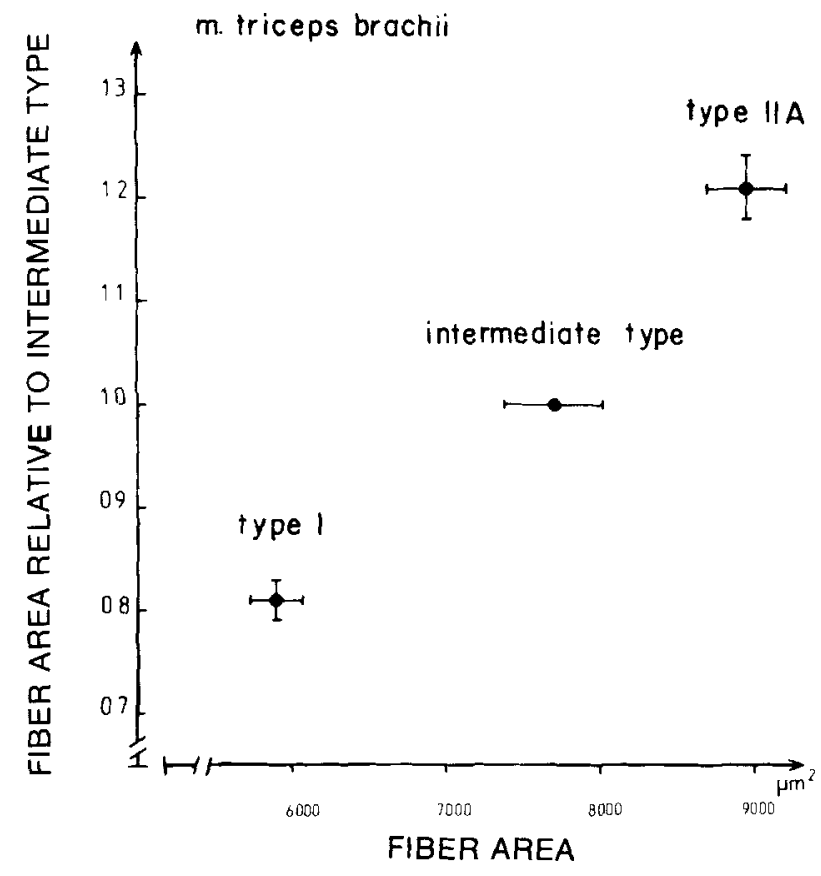

Figure 4. Cross-sectional area of types 1,2A, and intermediate fibers in 5 subjects. The values in the ordinate are mean values for the type 1 or type 2 area divided by the intermediate type area, and the values in the abscissa are absolute fiber type areas. (Means \pm SEM.)

other fiber types in 5 samples from 2 subjects. In these samples, the type 2B fiber areas did not differ from that of the intermediate fibers $\left[7,160 \mu \mathrm{m}^{2}\right.$ $(3,880$ to 8,830$)$ vs $6,110 \mu \mathrm{m}^{2}(4,200$ to 7,500$)$ n.s.].

Muscle Fiber Morphology. Eleven cases of longitudinally divided muscle fibers were found in a close examination of serially cut biopsy sections. They were evenly distributed among the 5 sampling sessions, including pretraining, and were seen in 5 of 6 subjects. Six of the cases were observed in the VL and the other 5 in the TB. Type 1 and type 2A fibers were affected to about the same degree. There were no obvious signs of fiber type grouping or atrophy. The mean fiber areas remained virtually unchanged during the experimental period. Nor was there any obvious increase in fiber size variability.

\section{DISCUSSION}

The main results of this training study were that the proportion of type 2 fibers decreased in the previously untrained arm muscles, while there was a concomitant increase in intermediate fibers. Even after a detraining period of 33 weeks, the percentage of type 2 fibers in the arms still had not reverted to the pretraining level, and intermediate fibers were still found in 5 out of the 6 subjects. No similar change was seen in the postural, and therefore previously better trained, leg muscles.

There is evidence suggesting that the intermediate fiber is in a state of transition into either type 1 or type 2 . Thus, it has been shown that myofibrillar ATPase intermediate fibers have contraction times between those of type 1 and 2 fibers (rat), ${ }^{27.28}$ and contain both fast and slow myosin (rabbit, human). ${ }^{4,31}$ Furthermore, intermediate fibers have been seen in connection with the transformation from type 2 to type 1 fibers in rat muscle in response to endurance training ${ }^{34}$ and rapid growth, ${ }^{28}$ as well as in rabbit muscle subjected to long-term electrical stimulation. ${ }^{37}$

That the intermediate fiber in fact represents a transitional stage is in the present study supported by the following: (l) a decrease in the percentage of type 2 fibers; (2) the intermediate fibers displayed immunohistochemical staining indicative of both fast- and slow-type myosin; and (3) the areas of the intermediate fibers were in between the areas of type 1 and type $2 \mathrm{~A}$. The staining of the training induced intermediate fibers toward both fast- and slow-type myosin in this study was the same as previously described for intermediate fibers. ${ }^{4}$ The intermediate size of the intermediate fibers is in agreement with the size reported for the intermediate fibers in human masseter muscle. ${ }^{40}$ Furthermore, it is in concordance with the decrease in fiber area in connection with the transformation from fiber type 2 to intermediate and/or type 1 fibers as a result of long-term electrical stimulation, ${ }^{7,37,43}$

However, if the intermediate fibers in the present study are signs of fiber type transformation, their failure to regress after a half year of detraining may seem strange, since total fiber type reconversion after discontinuation of long-term electrical stimulation of rabbit fast muscle occurs within 10 to 12 weeks. ${ }^{42}$ During the 33 weeks of detraining, however, the oxidative and glycolytic staining pattern of the intermediate fibers changed from one resembling the type 1 fibers to one more nearly resembling the type 2 fibers. This change may be indicative of an ongoing reconversion or a sign of a reconversion to come. The discrepancy between our results with respect to retransformation and those of Salmons ${ }^{42}$ may be due in part to the circumstances during detraining. In Salmons' study ${ }^{42}$ the rabbits were kept under the sedentary conditions of an animal colony during detraining, whereas in the present study the subjects made daily use of their arms in a rather uncontrolled 


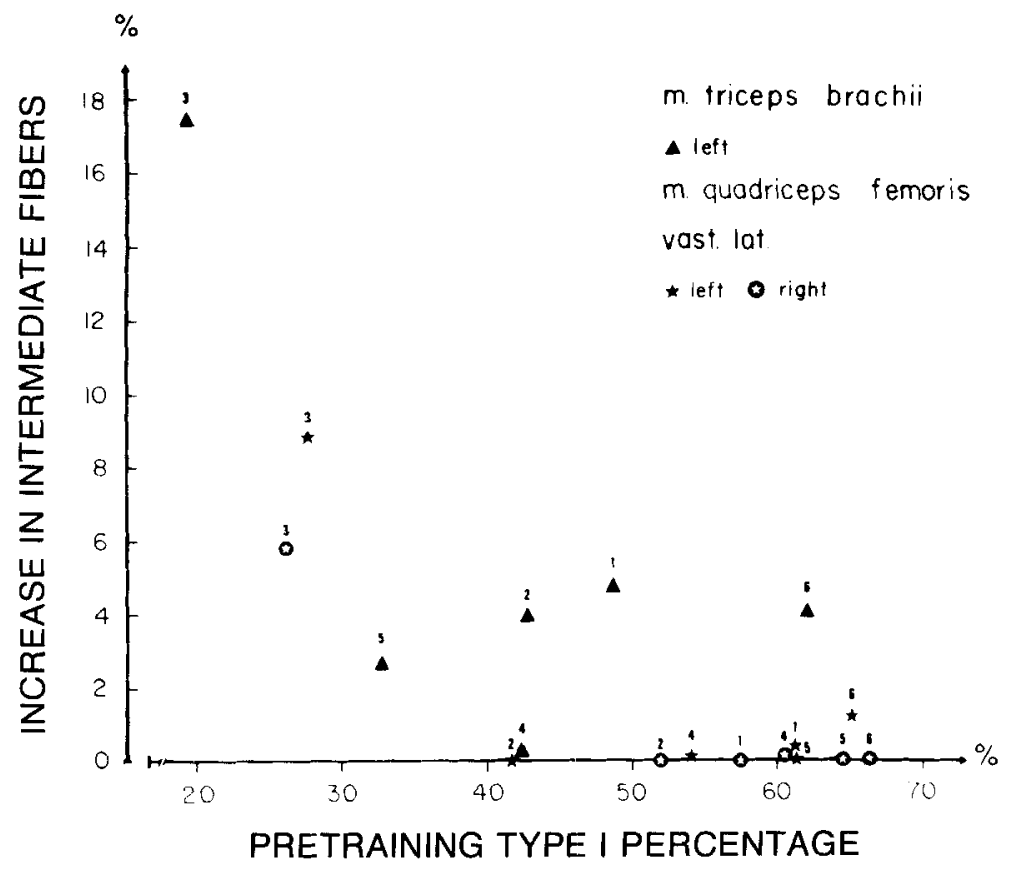

Figure 5. The increase in intermediate fibers during training (the values at week 3 and week 8 compared to pretraining with the highest value chosen) (ordinate), in relation to the pretraining percentage of type 1 fibers (abscissa). Each number represents one subject.

fashion. The discrepancy may also be due to differences in the rate of myosin turnover in humans and rabbits.

Theoretically, an explanation for the appearance of intermediate fibers during training and detraining could be that the repeated biopsy sampling caused nerve damage followed by reinnervation, a condition that has been suggested as the cause of increases in the number of intermediate fibers. ${ }^{28,33}$ This possibility cannot be ruled out, although it seems unlikely since there were no signs normally indicative of nerve damage, fiber injuries, or reinnervation, such as atrophy, myotubes, or type grouping. ${ }^{26,32,39}$ Furthermore, there was no obvious increase in the variability of fiber areas, and the mean fiber type areas remained virtually unchanged. The few cases of divided type 1 and type $2 \mathrm{~A}$ muscle fibers seen in both the VL and TB probably represent normal structural features as have been described for rat muscle, ${ }^{16}$ since they also were observed prior to training.

Kugelberg ${ }^{28}$ pointed out that intermediate fibers may either occur at the level of a single muscle fiber or an entire motor unit. The first situation was noted in less than $1 \%$ of the fibers in otherwise histochemically uniform units and was regarded as probably being due to a subterminal sprout. ${ }^{29}$ This may even be the origin of the small number of intermediate fibers normally seen in humans. In the latter situation, when entire motor units are histochemically intermediate, Kugelberg suggested that the cause must be due to altered neural influence. The aforementioned long-term stimulation studies of animal muscle suggest that histochemical fiber types are determined by the motor neuron firing rate. ${ }^{43}$ Therefore, the question to be answered in order to explain the occurrence of intermediate fibers in this study might be whether or not it is possible, with training, to change the discharge rate of the motor neurons innervating type 2 muscle fibers to an extent which induces fiber type alteration. To our knowledge, this matter has never been investigated. However, it is evident that the minimal and maximal firing rates of motor units, ${ }^{20,21,48}$ as well as the sequence of recruitment, ${ }^{19,20,48}$ may be affected by the afferent input. This indicates, at least, that motor unit electrical activity and activation does not depend solely on the unit's intrinsic properties but leaves open the question of the possible influence of physical training. Another conceivable explanation for the increase in intermediate fibers could be that training as such induced limited collateral sprouting. This has been found in rats subjected to treadmill running. ${ }^{41}$

Irrespective of this discussion, it seems reasonable to assume that a prerequisite for fiber transformation from fast to slow is involvement of the fast-twitch fibers in a type of activity in which the slow muscle fiber type is normally superior. This may be brought about, for example, when endurance exercise performed by a muscle demands greater tension than that which can be developed by the muscle's total type 1 fiber population alone, 
and following whole or partial glycogen depletion of the type 1 fibers, for example, after prolonged submaximal exercise. ${ }^{38}$ In the present study, different findings indicate that the total tension development capacity of the type 1 fibers, in relation to the work load, was more likely to be insufficient in the TB than was the case for the capacity of the type 1 fibers in the VL. (1) The subjects clearly experienced greater relative exertion in their arms, (2) the $10.8 \%(-0.2$ to 29.9$)$ smaller proportion of type 1 fibers in the TB compared to the VL, and (3) the relatively smaller type 1 fibers in the TB compared to the VL. The fact that the number of intermediate fibers in the triceps increased considerably more $(17.4 \%)$ in one subject with a very small percentage of type 1 fibers (19.9\%) lends support to this explanation. The same subject also had a small proportion of type 1 fibers in his legs, and was the only subject in whom an increased percentage of intermediate fibers was found in the legs (Fig. 5). The different initial training status of the two muscles may further explain the different response to training. The pretraining capillary supply and oxidative enzyme activities were markedly greater in the VL than in the TB. Thus, more rapid exercise-induced glycogen depletion in the $\mathrm{TB}$ can be expected (for discussion, see reference 23), resulting in earlier and/or more extensive involvement of type 2 fibers in the development of tension. Furthermore, the nature of the work performed by the two muscles differed. The arms performed dynamic work almost exclusively, whereas the leg work also comprised isometric-eccentric contractions.

In conclusion, it appears likely that a limited transformation between fiber types 2 and 1 may occur physiologically and that the intermediate fiber type constitutes a transitional phase of this transformation. However, the question of whether more extensive transformation may occur, leading to the extreme fiber type distributions seen in athletes, cannot be answered by the present study.

\section{REFERENCES}

1. Andersen P: Capillary density in skeletal muscle of man. Acta Physiol Scand 95:203-205, 1975.

2. Andersen P, Henriksson J: Training induced changes in the subgroups of human type II skeletal muscle fibres. $J$ Physiol (Lond) 270:677-690, 1977.

3. Bergström J: Muscle electrolytes in man. Scand J Clin Lab Invest [Suppl] 68:1-110,1962.

4. Billeter R, Weber H, Lutz H, Howald H, Eppenberger HM, Jenny $E$ : Myosin types in human skeletal muscle fibres. Histochemistry 65:249-259, 1980.

5. Brooke M, Kaiser K: Muscle fiber types: How many and what kind. Arch Neurol 23:369-379, 1970.

6. Brooke M, Kaiser K: Three "myosin ATPase" systems: The nature of their $\mathrm{pH}$ lability and sulfhydryl dependence. $J$ Histochem Cytochem 18:670-672, 1970.

7. Brown M, Cotter M, Hudlická O, Vrbová G: The effects of different patterns of muscle activity on capillary density, mechanical properties and structure of slow and fast rabbit muscles. Pfluegers Arch 361:241-250, 1976.

8. Costill DL, Daniels J, Evans W, Fink W, Krahenbuhl G, Saltin B: Skeletal muscle enzymes and fibre composition in male and female athletes. J Appl Physiol 40:149-154, 1976.

9. Dubowitz V, Brooke M: Muscle Biopsy: A Modern Approach. WB Saunders, London, 1973, pp 50-51.

10. Edström L, Torlegård K: Area estimation of transversely sectioned muscle fibres. $Z$ Wissenschaftliche Mikroskopie $\mathrm{Mi}$ kroskopische Technik 69:166-178, 1968-1969.

11. Eisen A, Karpati G, Carpenter S: Reserpine induced alteration of physiological properties and histochemical fibre types in rat skeletal muscle. Exp Neurol 46:554-565, 1975.

12. Engel $W$ : The essentiality of histo- and cyto-chemical studies of skeletal muscle in the investigation of neuromuscular disease. Neurology (Minneap) 12:778-794, 1962.

13. Eriksson BO, Gollnick PD, Saltin B: Muscle metabolism and enzyme activities after training in boys 11-13 years old. Acta Physiol Scand 87:231-239, 1972.

14. Gollnick P, Armstrong RB, Saltin B, Saubert CW IV, Sem- browich WL, Shepherd RE; Effect of training on enzyme activity and fibre composition of human skeletal muscle. $J$ Appl Physiol 34:107-111, 1973.

15. Gollnick P, Armstrong RB, Saubert CW IV, Piehl K, Saluin B: Enzyme activity and fibre composition in skeletal muscle of untrained and trained men. I Appl Physiol 33:312-319, 1972.

16. Gollnick P, Timson B, Moore R, Riedy M: Muscular enlargement and number of fibers in skeletal muscles of rats. J Appl Physiol 50:936-943, 1981.

17. Gomori G: The distribution of phosphatase in normal organs and tissues. J Cell Comp Physiol 17:7 1-83, 1941.

18. Green $H$, Thomson J, Daub W, Houston M, Ranney D: Fiber composition, fiber size, and enzyme activities in vastus lateralis of elite athletes involved in high intensity exercise. Eur J Appl Physiol 41:109-1 18, 1979.

19. Grimby L, Hannerz J: Recruitment order of motor units on voluntary contraction: Changes induced by proprioceptive afferent activity. $\int$ Neurol Neurosurg Psychiatry 31:565$573,1968$.

20. Grimby L, Hannerz J: Disturbances in voluntary recruitment order of low and high frequency motor units on blockades of proprioceptive afferent activity. Acta Physiol Scand 96:207-216, 1976 .

21. Hannerz J, Grimby L: The afferent influence on the voluntary firing range of individual motor units in man. Muscle Nerve 2:414-422, 1979.

22. Havu M, Rusko H, Komi PV, Vos J, Vihko V: Muscle fibre composition, work performance capacity and training in Finnish skiers. Int Res Communications System 1:10, 1973.

23. Holloszy J, Booth F: Biochemical adaptations to endurance exercise in muscle. Annu Rov Physiol 18:273-291, 1976

24. Jansson E, Sjödin B, Tesch P: Changes in muscle fibre type distribution in man after physical training. A sign of tibre type transformation? Acta Physiol Scand 104:235-237, 1978.

25. Jaweed MM, Herbison GI, Ditunno JF: Myosin ATPase activity after strengthening exercise. $J$ Anat 124:371-381, 1977. 
26. Karpati G, Engel K: Type grouping in skeletal muscles after experimental reinnervation. Neurology (Minneap) $18: 447-455,1968$.

27. Kugelberg E: Histochemical composition, contraction speed and fatiguability of rat soleus motor units. J Neurnl Sci 20:177-198, 1973.

28. Kugelberg E: Adaptive transformation of rat soleus motor units during growth. Histochemistry and contraction speed. I Neurol Sci 27:269-289, 1976.

29. Kugelberg E, Edström L, Abbruzzese M: Mapping of motor units in experimentally reinnervated rat muscle. $J$ Neurol Neurosurg Psychiatry 33:319-329, 1970.

30. Lutz H, Ermini M, Jenny E, Bruggmann S, Joris F, Weber E: The size of the fibre populations in rabbit skeletal muscles as revealed by indirect immunofluorescence with antimyosin sera. Histochemistry 57:223-235, 1978.

31. Lutz H, Weber E, Billeter R, Jenny E: Fast and slow myosin within single skeletal muscle fibers of adult rabbits. Nature 281:142-144, 1979.

32. Morris CJ: Human skeletal muscle fibre type grouping and collateral re-innervation. I Neurol Neurosurg Psychiatry $32: 440-444,1969$.

33. Morris CJ: The significance of intermediate fibres in reinnervated human skeletal muscle. J Neurol Sci 11:123-136, 1970.

34. Müller W: Temporal progress of muscle adaptation to endurance training in hind limb muscles of young rats. A histochemical and morphometrical study. Cell Tissue Res 156:61-88, 1974 .

35. Novikoff AB, Shin W, Druckner J: Mitochondrial localization of oxidation enzymes: Staining results with two tetrazolium salts. J Biophys Biochem Cytol 9:47-61, 1961.

36. Padykula HA, Herman E: The specificity of the histochemical method for adenosine triphosphatase. J Histochem Cytochem 3:170-195, 1955.

37. Pette D, Mülles $W$, Leisner E, Vrbová G: Time dependent effects on contractile properties, fibre population, myosin light chains and enzymes of energy metabolism in intermittently and continuously stimulated fast twitch muscle of the rabbit. Pfluegers Arch 364:103-112, 1976.

38. Piehl K: Glycogen storage and depletion in human skeletal muscle fibres, Acta Physiol Scand [Suppl] 402:1-33, 1974
39. Riley D: Histochemical changes in ATPase activity during regeneration of adult skeletal muscle fibers. Exp Neurol 41:690-704, 1973.

40. Ringqvist M: Fiber types in human masticatory muscles. Relation to function. Scand J Dent Res 82:333-355, 1974.

41. Roy R, Tweedle C, Stephens K, Ho K: Alterations in terminal innervation ratios in sprint and endurance trained rats. Med Sci Sports 10:67, 1978, abstract.

42. Salmons S: Functional adaptation in skeletal muscle. Trends Neurosci 3:134-137, 1980.

43. Salmons $\mathrm{S}$, Henriksson J: The adaptive response of skeletal muscle to increased use. Muscle Nerve 4:94-105, 1981.

44. Saltin B, Henriksson J, Nygaard E, Andersen P, Jansson E: Fibre types and metabolic potentials of skeletal muscles in sedentary man and endurance runners. Ann NY Acad Sci 301:3-29, 1977.

45. Saltin B, Nazar K, Costill DL, Stein E, Jansson E, Essén B, Gollnick PD: The nature of the training response: Peripheral and central adaptation to one-legged exercise. Acta Physiol Scand 96:289-305, 1976.

46. Schantz P, Henriksson J, Jansson E: Adaptation of human skeletal muscle to endurance training of long duration. Clin Physiol, to be published.

47. Snedecor G: Statistical Methods, 5th ed. Iowa State College Press, Ames, Iowa, 1956, p 251.

48. Stephens J, Garnett R, Buller N: Reversal of recruitment order of single motor units produced by cutaneous stimulation during voluntary muscle contraction in man. Nature 272:362-363, 1978.

49. Sternberger L: Immunocytochemistry, Chapter 5. New York, Wiley Medical, 1979.

50. Syrový I, Gutmann E, Melichna I: Effect of exercise on skeletal muscle myosin ATPase activity. Physiol Bohemoslov 21:633-638, 1972.

51. Thorstensson A, Hultén B, von Döbeln W, Karlsson J: Effect of strength training on enzyme activities and fibre characteristics in human skeletal muscle. Acta Physiol Scand 96:392--398, 1970.

52. Wattenberg LW, Leong JL: Effects of coenzyme Q10 and menadione on succinate dehydrogenase activity as measured by tetrazolium salt reduction. J Histochem Cytochem $8: 296-303,1960$. 